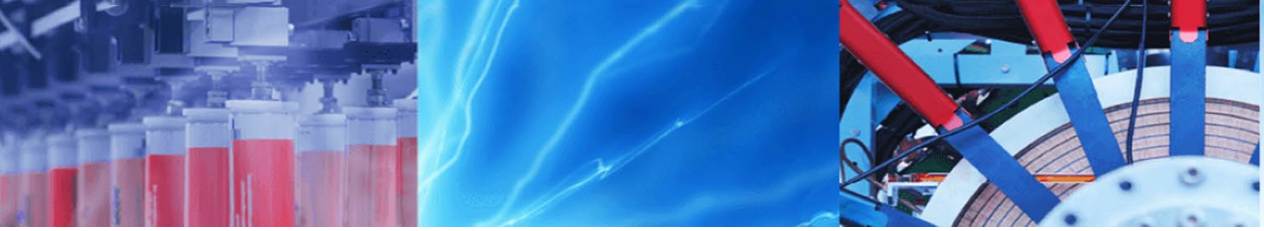

Short Communication

\title{
Emission enhancement in hybrid Tamm plasmon/photonic quasicrystal structure
}

\author{
Konstantin M. Morozov ${ }^{1,2}$ - Konstantin A. Ivanov $^{2} \cdot$ Aleksei V. Belonovskii $^{1} \cdot$ Elizaveta I. Girshova ${ }^{1}$
}

(c) Springer Nature Switzerland AG 2019

\begin{abstract}
We have theoretically demonstrated that Tamm plasmon structure with photonic quasicrystal instead of usual distributed Bragg reflector can support hybrid Tamm plasmon-like optical eigenstates. The model structure consists of a $\mathrm{SiO}_{2} / \mathrm{Ta}_{2} \mathrm{O}_{5}$ optical Fibonacci system with a thin silver layer on the top. $F_{7}$-based hybrid cavity was analyzed by means of calculation reflectivity spectrum and electric field profiles of the localized states. Also, evaluation of the modal Purcell factor for hybrid structure demonstrates significant enhancing of spontaneous decay rate for emitter coupled with Tamm plasmon-like states. The results can be used in fabrication of high-efficient organic-based light emission systems.
\end{abstract}

Keywords Plasmonics · Tamm plasmon · Photonic quasicrystal · Purcell effect

\section{Introduction}

Practical applications of plasmonic structures are limited due to absorption in metals (even noble ones) that lead to losses and ohmic heating [1]. In this case, new concepts and decisions of structures with metallic parts need to be found for the fabrication of plasmonic-based optoelectronic applications such as light sources, modulators and solar cells. In the last few years, there is growing interest to systems, that support Tamm plasmon (TP) [2]. Tamm plasmon is a novel electromagnetic state localized at the interface between the metal and the specially designed Bragg reflector. Operation in TP mode produces lower absorption losses due to electric field distribution properties. (TP state has electric field node in the metal layer that suppresses the absorption.) In addition, TP modes have some useful advantages in comparison with convenient surface plasmon [3]. Tamm plasmon is forming in both TE and TM polarizations. Its dispersion lies inside the light cone that allows exciting Tamm plasmon state directly, without any gratings or prisms. Recently, single-photon emission [4] and lasing [5] in Tamm plasmon-based systems were demonstrated. As well as promising sensor designs $[6,7]$ with Tamm plasmon were shown.

Photonic quasicrystals (PQC) are a class of photonic structures take place between ordered and fully disordered photonic systems [8]. PQC has long-range order but lack of periodicity. Such systems have peculiar optical properties like the fractal density of photonic states and are determined by this light localization even in 1D case [9-11]. Also, recent studies demonstrate the non-trivial topological properties of PQC structures [12]. Combination of metal-based systems with photonic crystals can lead to fascinating phenomena $[13,14]$. Recently, topological properties of the TP-like state formed in a 1D photonic quasicrystal were studied [15].

This paper is aimed at a theoretical study of the hybrid Tamm plasmon-photonic quasicrystal cavity optical properties and light emission enhancement phenomenon in this system.

Konstantin M. Morozov, morzconst@gmail.com | 'St. Petersburg Academic University, 8/3 Khlopina St., Saint Petersburg, Russia 194021. ${ }^{2}$ ITMO University, 49 Kronverksky Pr., Saint Petersburg, Russia 197101. 


\section{Hybrid photonic quasicrystal structure with Tamm plasmon}

Usual Tamm plasmon (TP) structure consists of a distributed Bragg reflector (DBR) and the metal layer on the top of it (Fig. 1a). In our study, Tamm plasmon cavity was constructed by two base layers $\mathrm{A}: \mathrm{SiO}_{2}$ (silicon oxide) and $\mathrm{B}: \mathrm{Ta}_{2}$ $\mathrm{O}_{5}$ (tantalum pentoxide) and the silver layer. These materials are common in modern photonic microcavity fabrication process due to its optical properties. The thickness of the silver layer was chosen as $50 \mathrm{~nm}$, dielectric layers thickness corresponded to quarter-wavelength: $d_{\mathrm{A}, \mathrm{B}}=\lambda_{0} / 4 n_{\mathrm{A}, \mathrm{B}}$. Refractive indexes of dielectric materials $\left(n_{A, B}\right)$ were taken from [16].

The Fibonacci optical lattice is the simplest photonic quasicrystal (PQC) system. As well as the DBR, the Fibonacci lattice is a sequence of layers of two types $(A, B)$, but alternation, in this case, is not periodic. The Fibonacci lattice of order $F_{i+1}$ can be constructed by the recurrent rule

$F_{i+1}=\left\{F_{i-1}, F_{i}\right\}$

with initial values $F_{0}=\mathrm{A}$ (single $\mathrm{SiO}_{2}$ layer) and $F_{1}=\mathrm{B}$ (single $\mathrm{Ta}_{2} \mathrm{O}_{5}$ layer). Examples of the first seven Fibonacci sequences are shown in Table 1. The scheme of the hybrid TP structure with the Fibonacci optical lattice instead of DBR is shown in Fig. $1 b$.

\section{Results and discussion}

At first, we will briefly discuss optical properties cavities dielectric component-1D photonic crystal (DBR) and quasicrystal (optical Fibonacci lattice). In 1D PC photonic band gap (PBG), formation is expected - the area with almost full light reflection. Formation of the single PBG in 1D periodic DBR structure is demonstrated in Fig. 2a by the crimson line. Reflectivity spectra are centred at a characteristic frequency, governed by properties of $A$ and $B$ materials:

$\omega_{0}=\frac{\pi c}{n_{\mathrm{A}} d_{\mathrm{A}}+n_{\mathrm{B}} d_{\mathrm{B}}}$

in case of chosen model parameters $\left(d_{A}=70 \mathrm{~nm}\right.$ and $d_{B}$ $=47.2 \mathrm{~nm}$ ), characteristic energy will be $2 \mathrm{eV}$. Due to the fractal density of photonic states of the optical Fibonacci lattices [10], the reflectivity spectrum of PQC's number of PGB's will be higher than in the periodic case (see Fig. 2b-f). Furthermore, increasing the lattice order leads to increase in PBG's number. It can be seen that band gap's positions are centred at $\hbar \omega_{0}$ energy in each case.

To form the TP cavity structure, it is needed to place a thin metal layer on the top of the DBR. Then, the resonance condition inside PBG on the wave phase will be fulfilled and the electromagnetic TP eigenstate will appear. The TP resonance with the spectral position inside the PBG region corresponds to sharp deep in the reflectivity spectrum (see the black curve in Fig. 2a). If instead of the 1D photonic crystal (DBR), the quasicrystalline system will be the main part of the cavity picture will be changed: the multiple TPlike states will appear (see the black curve Fig. 2b-f). Each notable deep in the hybrid cavity reflectivity spectrum corresponds to the TP-like state. When the photonic structure

Table 1 First seven Fibonacci sequences layer configuration

\begin{tabular}{ll}
\hline Order & Configuration \\
\hline$F_{0}$ & $\mathrm{~A}\left(\mathrm{SiO}_{2}\right)$ \\
$F_{1}$ & $\mathrm{~B}\left(\mathrm{Ta}_{2} \mathrm{O}_{5}\right)$ \\
$F_{2}$ & $\mathrm{AB}$ \\
$F_{3}$ & $\mathrm{BAB}$ \\
$F_{4}$ & $\mathrm{ABBAB}$ \\
$F_{5}$ & BABABBAB \\
$F_{6}$ & $\mathrm{ABBABBABABBAB}$ \\
$F_{7}$ & BABABBABABBABBABABBAB \\
\hline
\end{tabular}

Fig. 1 a Scheme of conventional Tamm plasmon system. b Scheme of hybrid Tamm plasmon quasicrystal structure

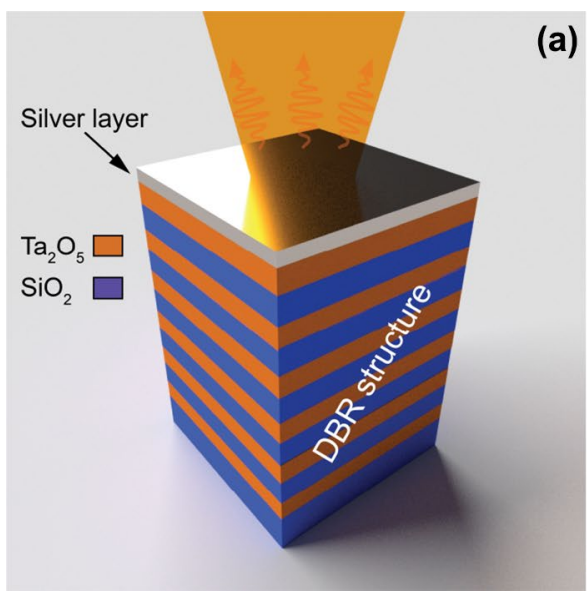

(a)

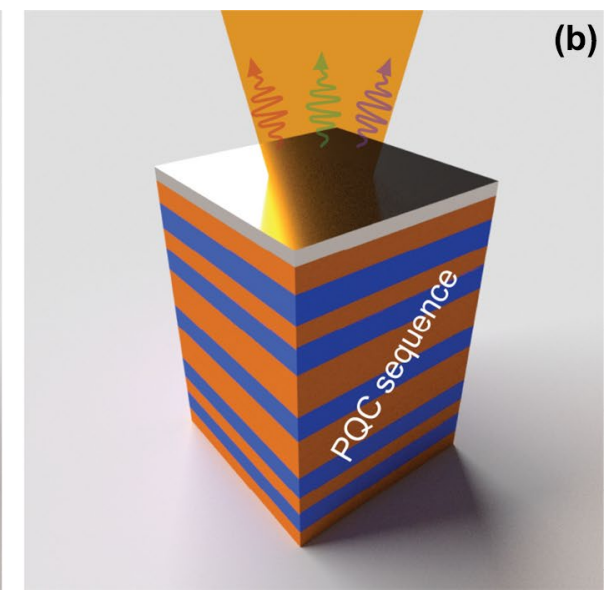


Fig. 2 Reflectivity spectra of DBR (a) and optical Fibonacci lattices $F_{5}(\mathbf{b}), F_{6}(\mathbf{c}), F_{7}(\mathbf{d}), F_{8}$ (e), $F_{9}(\mathbf{f})$-crimson curve. The black curve shows the reflectivity spectrum of each TP structure, formed by stack with the metal layer (a)

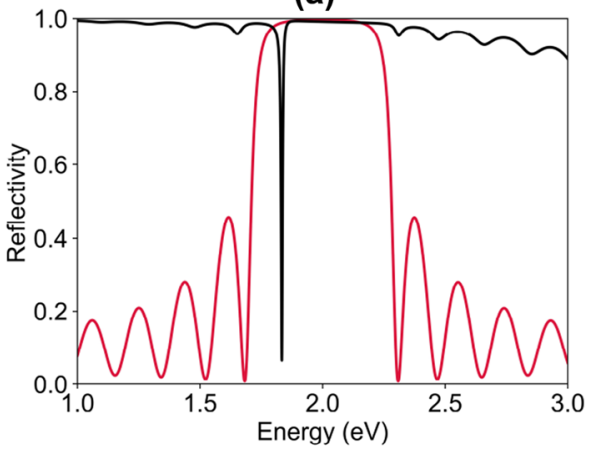

(c)

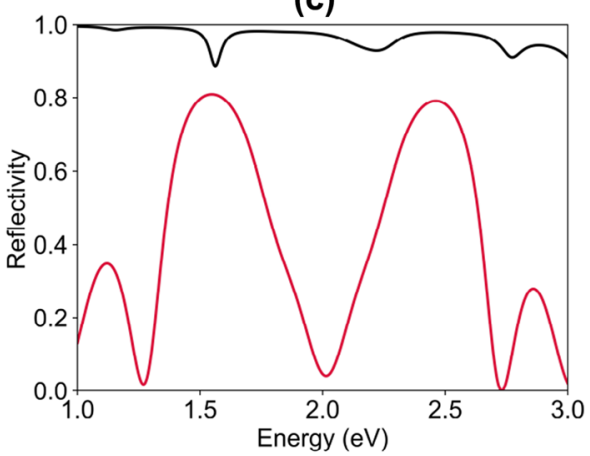

(e)

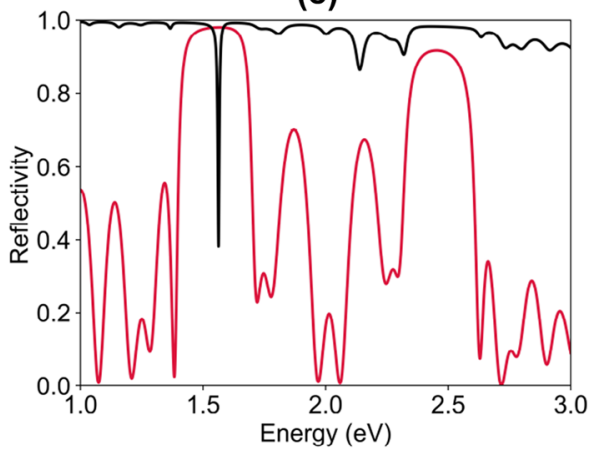

(b)

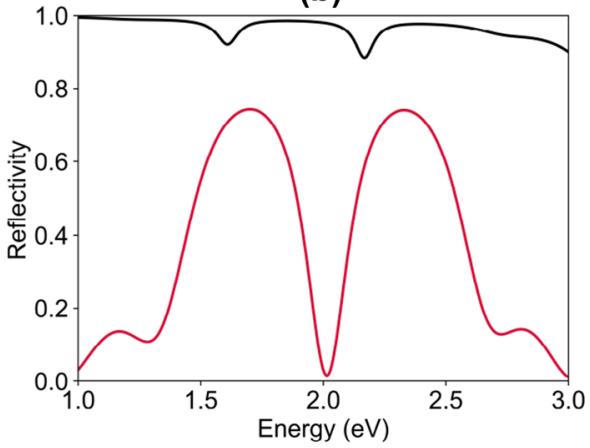

(d)

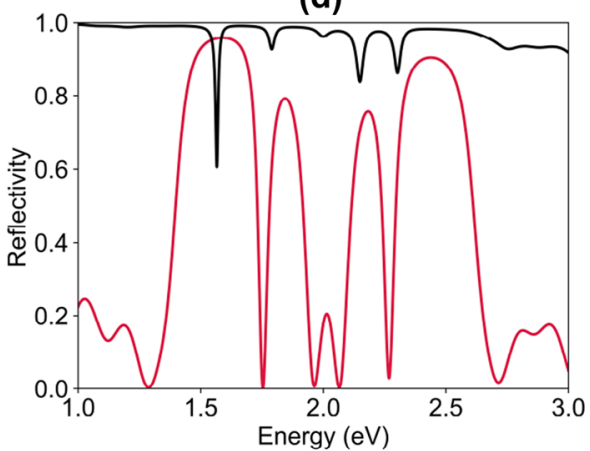

(f)

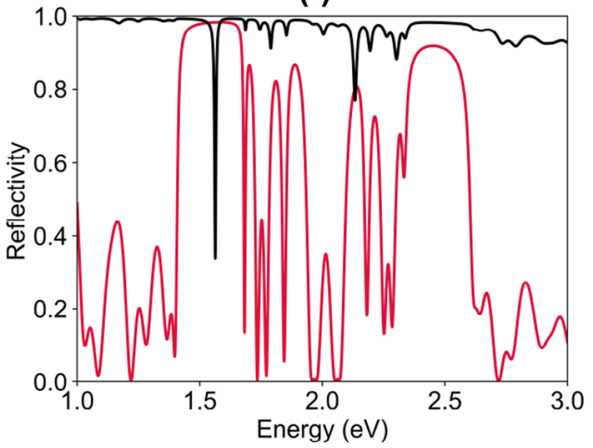

has a number of PBG's, each PBG has a spectral area where phase condition is satisfied. In the case of $F_{7}$-based cavity (Fig. $2 \mathrm{~d}$ ), there are five notable deeps ( $\hbar \omega_{1}=1.56 \mathrm{eV}$, $\left.\hbar \omega_{2}=1.79 \mathrm{eV}, \hbar \omega_{3}=2 \mathrm{eV}, \hbar \omega_{4}=2.14 \mathrm{eV}, \hbar \omega_{5}=2.3 \mathrm{eV}\right)$ in the spectrum that corresponds to the eigenstates.

For the better understanding of this hybrid state properties, electric field distribution in case of $F_{7}$-based cavity was calculated. Distributions of the TP-like states electric field for case of TE polarization are shown in Fig. 3. Figure $3 a$ shows the numbering of the five noticeable features in the reflectivity spectrum. As you can see, the most notable resonance deep corresponds to lesser energy $(1.56 \mathrm{eV})$. Electric field profile of this state (Fig. 3b) looks almost similar to usual TP state - the field is maximum in the first layer of structure and its value monotonically decays with the increase in distance from the metal. On the other hand, field profiles of the other TP-like states are more peculiar. Despite this, in each case profile overlap with the cavity region (usually the first dielectric layer borders with the metal-blue area in the figures) and has at least local maximum. To that end, instead of a single high-quality Tamm plasmon state, in hybrid photonic quasicrystal-Tamm plasmon system, we have set of TP-like states, in the wide spectral area that overlaps with the main cavity region (dielectric layer bordering with the metal).

For the potential device applications, it is important to understand how such hybrid cavity environment may influence on the decay rate of the emitter placed inside the active layer. By using S-quantization formalism [17], modal Purcell coefficient dependence on the emission energy and the angle was calculated (Fig. 4) for the dipole placed at the edge of cavity region. Figure 4 highlights spectral regions where significant enhancement of the dipole spontaneous decay rate takes place. To evaluate Purcell coefficient in a well-known meaning modal Purcell coefficient, it is needed to be averaged by 
Fig. 3 a Reflectivity spectrum of $F_{7}$-based cavity (black curve). Numbers indicate TP-like states. b-f Normalized $|E|^{2}$ distribution of the TP-like states in structure (blue curve). The red area represents the silver layer, and the blue area represents the first dielectric layer (a)

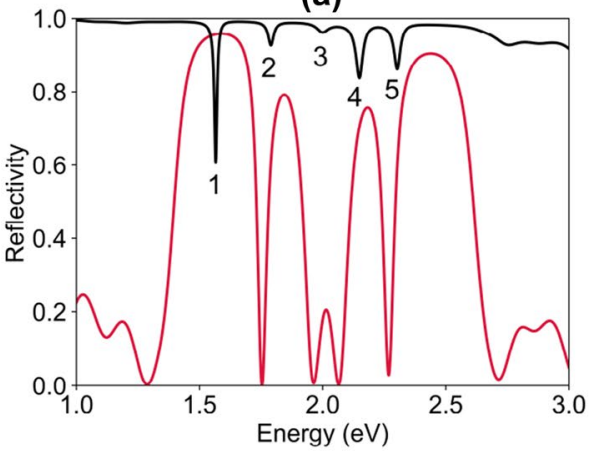

(c)

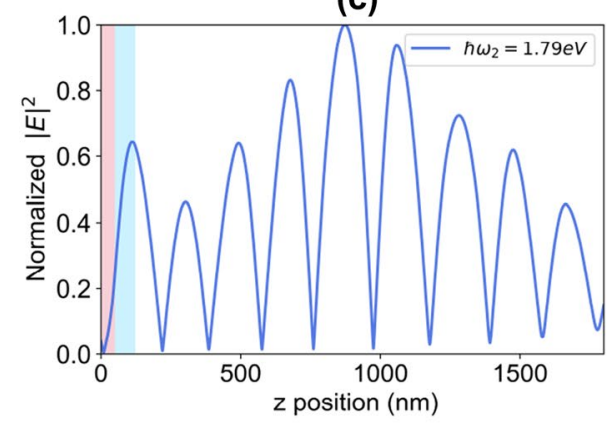

(e)

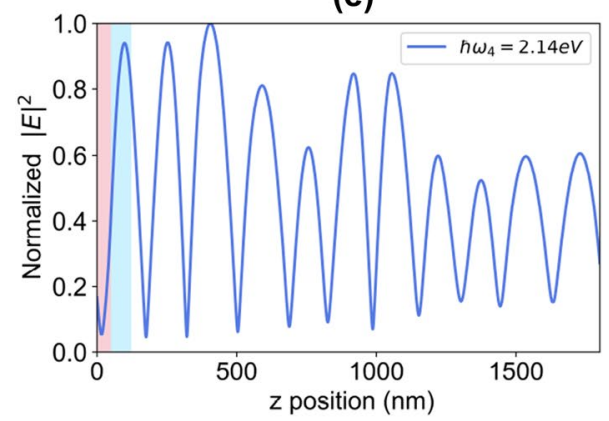

(b)

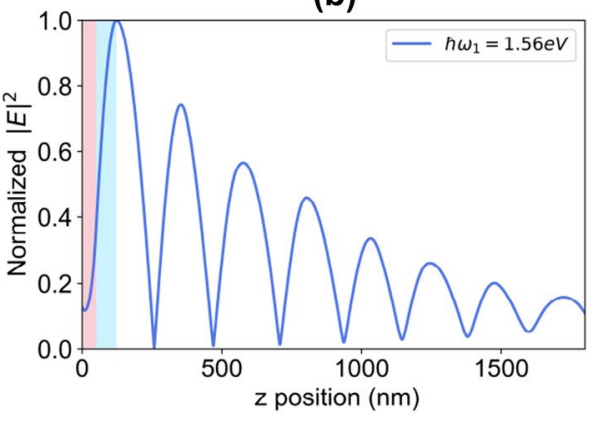

(d)

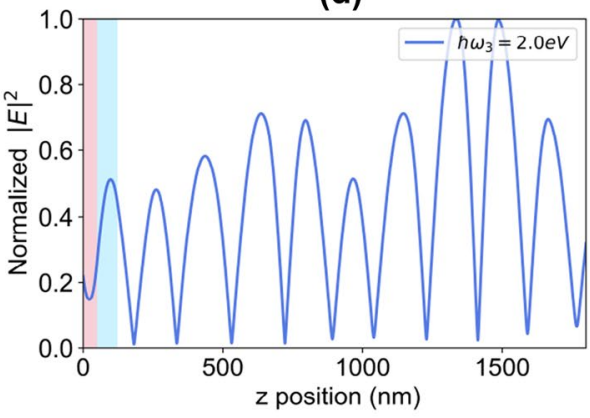

(f)

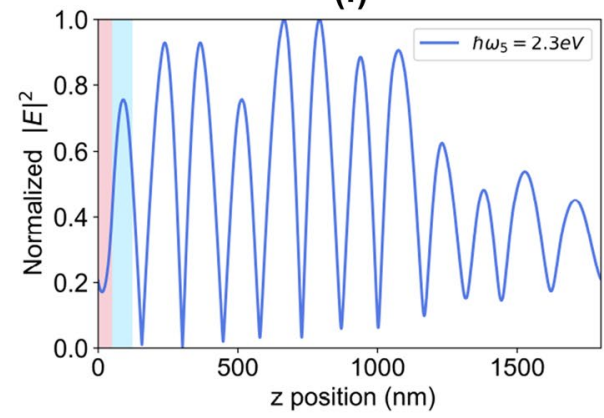

all angles and polarizations. Dispersion of TP-like states is almost similar to usual TP state dispersion. Despite the fact that area with the highest modal Purcell factor (up to 47$)$ falls on the lowest energy state $\left(\hbar \omega_{1}=1.56 \mathrm{eV}\right)$ and state with energy $\hbar \omega_{4}=2.14 \mathrm{eV}$ (both has closest to usual TP state E-field profile (Fig. 3b, e)), each other mode can noticeably enhance emission efficiency of the point dipole. However, averaging of modal Purcell factor, taking into account material dispersion and absorption in dielectric media, will lead in the experiment to the moderate values of the Purcell coefficient like shown in works $[3,4,18]$. Nevertheless, it is known that some organic light-emitting molecules have a wide emission spectrum (up to $1 \mathrm{eV}$ ) and Purcell enhancement in the wide spectral region can be useful in such systems. We think that hybrid photonic quasicrystal-Tamm plasmon cavity will become a promising part of high-efficient photonic devices based on organic materials.

\section{Conclusions}

Hybrid one-dimensional photonic quasicrystal-Tamm plasmon cavity was considered. The results demonstrate that such systems support multiple Tamm plasmon-like hybrid states in the wide spectral region. Evaluation of the modal Purcell factor for the hybrid structure was carried out. Significant enhancing of spontaneous decay rate for emitter coupled with Tamm plasmon-like states was demonstrated. The results show that hybrid TP-like states can be used in the increase in the organic lightemitting materials efficiency and in the complex light emission systems. 


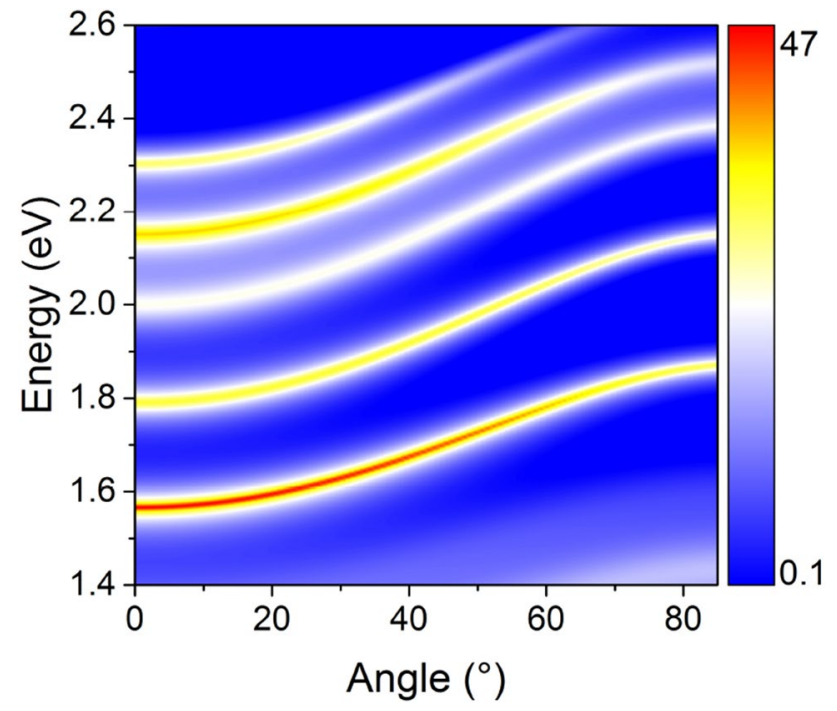

Fig. 4 Modal Purcell factor distribution calculated for $F_{7}$-based hybrid cavity for emitter placed in the edge between the first and second dielectric layer (in case of TE polarization)

Acknowledgements The work was supported by the Grant of Minobrnauki No. 16.9789.2017/BCh.

\section{Compliance with ethical standards}

Conflict of interest The authors declare that they have no conflict of interest.

\section{References}

1. Khurgin JB (2015) How to deal with the loss in plasmonics and metamaterials. Nat Nanotechnol 10(1):2. https://doi. org/10.1038/nnano.2014.310

2. Kaliteevski MA, Lazarenko AA, Il'inskaya ND, Zadiranov YM, Sasin ME, Zaitsev D, Mazlin VA, Brunkov PN, Pavlov SI, Egorov AY (2015) Experimental demonstration of reduced light absorption by intracavity metallic layers in Tamm plasmon-based microcavity. Plasmonics 10(2):281. https://doi.org/10.1007/s1146 8-014-9806-0

3. Gubaydullin AR, Symonds C, Bellessa J, Ivanov KA, Kolykhalova ED, Sasin ME, Lemaitre A, Senellart P, Pozina G, Kaliteevski MA (2017) Enhancement of spontaneous emission in Tamm plasmon structures. Sci Rep 7(1):9014. https://doi.org/10.1038/ s41598-017-09245-7

4. Gazzano O, Michaelis De Vasconcellos S, Gauthron K, Symonds C, Voisin P, Bellessa J, Lemaître A, Senellart P (2012) Single photon source using confined Tamm plasmon modes. Appl Phys Lett 100(23):232111. https://doi.org/10.1063/1.4726117
5. Symonds C, Lheureux G, Hugonin JP, Greffet JJ, Laverdant J, Brucoli G, Lemaitre A, Senellart P, Bellessa J (2013) Confined Tamm plasmon lasers. Nano Lett 13(7):3179. https://doi.org/10.1021/ nl401210b

6. Auguié B, Fuertes MC, Angelomé PC, Abdala NL, Soler Illia GJ, Fainstein A (2014) Tamm plasmon resonance in mesoporous multilayers: toward a sensing application. ACS Photon 1(9):775. https://doi.org/10.1021/ph5001549

7. Zhang C, Wu K, Giannini V, Li X (2017) Tamm plasmons, hot electrons, photodetector, photoresponsivity, surface plasmons. ACS Nano 11(2):1719. https://doi.org/10.1021/acsnano.6b07578

8. Vardeny ZV, Nahata A, Agrawal A (2013) Optics of photonic quasicrystals. Nat Photon 7(3):177. https://doi.org/10.1038/nphot on.2012.343

9. Gellermann W, Kohmoto M, Sutherland B, Taylor PC (1994) Localization of light waves in Fibonacci dielectric multilayers. Phys Rev Lett 72(5):633. https://doi.org/10.1103/PhysRevLett.72.633

10. Kaliteevski MA, Nikolaev VV, Abram RA, Brand S (2002) Bandgap structure of optical Fibonacci lattices after light diffraction. Opt Spectrosc 91(1):109. https://doi.org/10.1134/1.1388332

11. Morozov K, Ivanov K, Gubaydullin A, Kaliteevski M (2017) Purcell effect in one-dimensional photonic quasicrystals. Opt Spectrosc 122(2):235. https://doi.org/10.1134/S0030400X17020199

12. Verbin $M$, Zilberberg $O$, Lahini $Y$, Kraus $Y E$, Silberberg $Y$ (2015) Topological pumping over a photonic Fibonacci quasicrystal. Phys Rev B Condens Matter Mater Phys. https://doi.org/10.1103/ PhysRevB.91.064201

13. Gallant AJ, Kaliteevski MA, Wood D, Petty MC, Abram RA, Brand S, Swift GP, Zeze DA, Chamberlain JM (2007) Passband filters for terahertz radiation based on dual metallic photonic structures. Appl Phys Lett. https://doi.org/10.1063/1.2800381

14. Swift GP, Gallant AJ, Kaliteevskaya N, Kaliteevski MA, Brand S, Dai D, Baragwanath AJ, lorsh I, Abram RA, Chamberlain JM (2011) Negative refraction and the spectral filtering of terahertz radiation by a photonic crystal prism. Opt Lett 36(9):1641. https://doi. org/10.1364/ol.36.001641

15. Shukla MK, Das R (2018) Tamm-plasmon polaritons in onedimensional photonic quasi-crystals. Opt Lett 43(3):362. https ://doi.org/10.1364/ol.43.000362

16. Rodríguez-de Marcos LV, Larruquert JI, Méndez JA, Aznárez JA (2016) Self-consistent optical constants of $\mathrm{SiO}_{2}$ and $\mathrm{Ta}_{2} \mathrm{O}_{5}$ films. Opt Mater Express 6(11):3622. https://doi.org/10.1364/ OME.6.003622

17. Morozov K, Girshova E, Gubaidullin A, Ivanov K, Pozina G, Kaliteevski M (2018) Different regimes of the Purcell effect in disordered photonic crystals. J Phys Condens Matter 30(43):435304. https://doi.org/10.1088/1361-648X/aae18c

18. Morozov K, Ivanov K, Selenin N, Mikhrin S, de Sa Pereira D, Menelaou C, Monkman A, Kaliteevski M (2018) Experimental study of spontaneous-emission enhancement in Tamm plasmon structures with an organic active region. Semiconductors 52(11):1420. https://doi.org/10.1134/S1063782618110155

Publisher's Note Springer Nature remains neutral with regard to jurisdictional claims in published maps and institutional affiliations. 